Article

\title{
Three-Dimensional Facial Asymmetry in Attractive and Normal People from Childhood to Young Adulthood
}

\author{
Chiarella Sforza $^{1, *}$, Alberto Laino ${ }^{2}$, Gaia Grandi ${ }^{1}$, Luca Pisoni ${ }^{1}$ and Virgilio Ferruccio Ferrario ${ }^{1}$ \\ 1 Laboratorio di Anatomia Funzionale dell’Apparato Stomatognatico, Dipartimento di Morfologia \\ Umana e Scienze Biomediche “Città Studi”, Università degli Studi di Milano, 20122 Milan, Italy; \\ E-Mails: Gaia.Grandi@gmail.com (G.G.); Luca.Pisoni@studenti.unimi.it (L.P.); \\ Virgilio.Ferrario@unimi.it (V.F.F.) \\ 2 Dental, Oral, and Maxillo-Facial Sciences, Section of Orthodontics, University Federico II, 80125 \\ Naples, Italy; E-Mail: Alberto.Laino@unina.it
}

* Author to whom correspondence should be addressed; E-Mail: Chiarella.Sforza@unimi.it.

Received: 1 October 2010 / Accepted: 19 October 2010 / Published: 9 November 2010

\begin{abstract}
We are currently investigating measurable esthetic characteristics in persons considered "attractive” by the media. Three-dimensional soft-tissue facial asymmetry was quantified in 380 attractive (148 males, 232 females) and 669 control (397 males, 272 females) healthy persons aged 4-30 years. The coordinates of 50 facial landmarks were collected by a computerized digitizer, and asymmetry computed. Soft-tissue facial asymmetries reduced as a function of age in all cases. Attractive children were more symmetric than control children, but the reverse was true for young adults. The effect of symmetry on attractiveness seems to change as a function of age.
\end{abstract}

Keywords: attractiveness; face; symmetry; three-dimensional

\section{Introduction}

Asymmetry is a common finding in both the craniofacial hard tissues, and in the facial appearance, where the soft tissue cover (muscles, skin, adipose tissue) may partly mask the underlying imbalances [1-9]. Asymmetry is also found in the movement of facial mimic muscles: in healthy subjects, facial expressions, and in particular spontaneous smile, are larger on the left- than on the right-side of the face [10-12]. 
Facial asymmetry is better appreciated using a three-dimensional approach that allows a direct measurement of the two facial halves $[6,7,13,14]$, because the visual, qualitative assessment is often insufficient. For instance, skeletal asymmetries of less than $3 \%$ are not clinically discernible $[5,15]$. If the points of ear insertion cannot be easily identified, facial depth may be of difficult visual evaluation [16]. Indeed, in the face all three spatial components (right-left, cranio-caudal, anteriorposterior) can be asymmetric. In particular, the facial lower third can be very asymmetric in the anteroposterior plane $[2,8]$, a finding neglected in the conventional two-dimensional photographic or radiographic views [1,5,17-19].

According to the current theories of evolutionary psychology, the esthetic assessment of adult faces depends on various combinations of averageness, symmetry, neoteny (babyness) and youthfulness, and sexual dimorphism [19-27]. Additionally, facial expression (happiness, excitement, arousal) and grooming (successful adaptation, status) concur to a global positive assessment [21].

Both natural and sexual selections seem to explain the various perceptions of attractiveness, with almost consistent ratings across sexes, ethnic groups and ages [13,20,24-26]. Recently, these investigations were extended also to children and adolescents [28-31]. Overall, the studies confirmed some of these theories: attractive children, female adolescents and adult women had faces with several characteristics of babyness: a large face with a relatively large forehead; relatively prominent cheekbones; reduced vertical development; full and prominent lips, and a more convex soft-tissue facial profile, than normal coetaneous persons [20,21,28-32]. In contrast, in post-pubertal adolescent boys and adult men, attractiveness seems to be positively influenced by facial markers of high testosterone levels (a signal of sexual dimorphism), with a relative increment of the facial lower third (more prominent chin, less prominent lips) [20,22,25,30,33].

In our laboratory, we are currently studying the three-dimensional facial characteristics of children, adolescents, adult males and females considered "attractive" by the general public [30-32]. Their facial soft tissues were measured with a non-invasive computerized instrument, several measurements obtained, and compared to those collected in healthy persons of the same sex, age and ethnicity, selected using criteria of dentofacial normality (Figure 1). The presence of measurable specific characteristics was assessed. If esthetically pleasing faces possess some kind of codified facial dimensions, angles and ratios, these measurements could be used by medical and dental practitioners for a better patient care [6,9,34]. Previous investigations performed on these attractive persons assessed the hypotheses of babyness/ youthfulness (found to explain a large part of facial attractiveness in children, adolescent girls, and adult women) and of sexual dimorphism (an effect found in adult women, in male adolescents, and in adult men) [30-32]. On no occasion, was data about their facial symmetry/ asymmetry assessed. 
Figure 1. Facial landmarks digitized on all subjects for subsequent morphometric calculations. Midline landmarks: tr, trichion; g, glabella; n, nasion; prn, pronasale; c', columella; sn, subnasale; ls, labiale superius; sto, stomion; li, labiale inferius; sl, sublabiale; pg, pogonion; me, menton. Paired (right and left side) landmarks: ex, exocanthion; en, endocanthion; os, orbitale superius; or, orbitale; ft, frontotemporale; chk, cheek; zy, zygion; t, tragion; al, alare; ac, nasal alar crest; itn, inferior point of the nostril axis; stn, superior point of the nostril axis; cph, crista philtri; ch, cheilion; go, gonion; pra, preaurale; sa, superaurale; pa, postaurale; sba, subaurale.

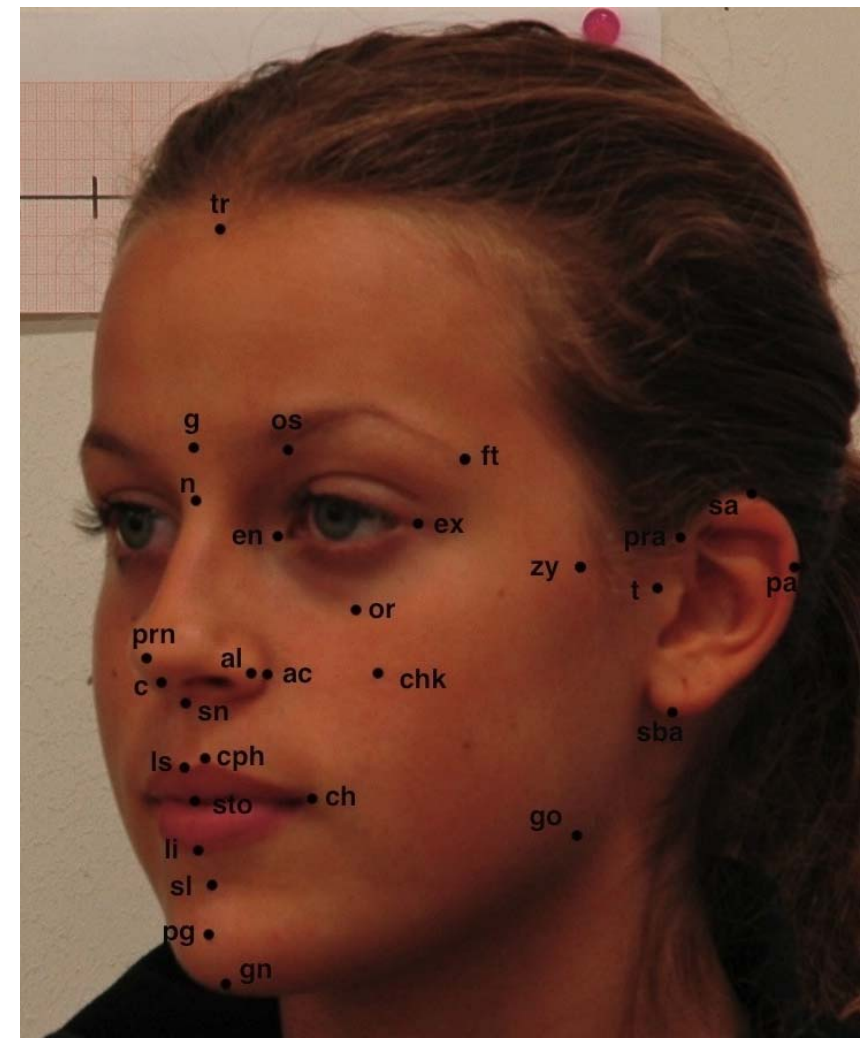

\subsection{Facial Symmetry and Attractiveness}

Asymmetry can be divided into antisymmetry, directional and fluctuating asymmetry. In antisymmetry, either side of the body could be dominant (for instance, handedness). Directional asymmetry is consistently dominant on one side (for instant, heart position), while fluctuating asymmetry relates to traits that are on average symmetric in a population. Fluctuating asymmetry is considered to result from unbalances in growth and development of the various structures, with a reduced capacity of the organism to buffer environmental disturbances and genetic stressors [4,13,14,19,26,35-37]. From this point of view, the assessment of fluctuating asymmetry may provide useful information about the combined action of developmental stress and individual capacity to react.

An increased fluctuating asymmetry has been related to higher morbidity and mortality, reduced fecundity, inferior locomotory performance $[13,35]$. In contrast, a reduced fluctuating asymmetry has been associated with increased attractiveness, both in the face and in the body $[13,19,21-24,27]$. 
Current explanations about the effect of facial symmetry on attractiveness can be summarized into three main aspects: symmetry is an important signal of good health and developmental stability; during visual inspection, symmetric faces are easier to scan and understand; symmetric faces are nearer to the average, prototype face [19,21-24,27]. Persons with symmetric faces and bodies suggest to the observer that they possess good genes, a good general health, and are more resistant to diseases and parasites. Symmetric faces become "honest" markers of genotype and phenotype quality: Symmetry reflects the individual ability to maintain a stable development notwithstanding the environmental stress, thus being the result of natural selection [4,13,19,21,23,24,26,36,38].

Despite these theories, not all investigators concord about a strict relationships between facial symmetry and attractiveness from one side, and facial asymmetry and disease (or altered development) from the other side [1,12,18,21,27,36,38]. For instance, Peck et al. [5] found that men and women considered attractive by the general public (beauty competition winners, professional models) had asymmetries in one or more craniofacial skeletal dimensions. Zaidel and Hessamian [36] found that both full and hemi-faces (only right or left side of the face) were rated similarly in terms of attractiveness. Shaner et al. [14] did not find an increase in soft-tissue facial asymmetry in syndrome affected individuals as compared to normal persons.

In our previous investigations, among the various analyzed parameters, the effect of facial symmetry on attractiveness was not considered. In the current study, we assessed the effect of facial symmetry/asymmetry on attractiveness by analyzing a wide group of attractive persons of both sexes ranging from four to 30 years of age, as well as a group of normal persons of the same age, sex and ethnic group. Their fluctuating soft-tissue facial asymmetry was quantitatively obtained from the three-dimensional coordinates of selected soft-tissue facial landmarks [14]. In a first analysis, linear distances and landmark coordinates were considered using a method previously developed in our laboratory, and that had been used to assess sex- and age- related variations in healthy people, as well as the effect of pathology and relevant treatment [3,8,39]. The method has been constructed considering the object symmetry of the human face [37]; it allows to separate the effect of selected landmarks to the total facial asymmetry, as recently underlined [40], and it has proved to supply clinically relevant information $[8,39]$.

Attractive persons were selected by casting agencies or by judges of beauty competitions, without any knowledge of the aims and scope of the current investigations (see details below). Similar protocols were followed by Peck et al. [5] and by Ing et al. [18] for two-dimensional hard- and softtissue craniofacial characteristics. This procedure contrasts with that followed in most studies about facial attractiveness, where the judgment was made on photographs (both original images, and composite or manipulated faces) of unselected persons [19,21-26,33,36,38].

\subsection{Selection of Attractive Persons}

The selection of attractive persons was made by independent judges, who were unaware of the scope of the investigation. Children and adolescents were selected by a commercial casting agency located in northern Italy, which was asked to provide white Caucasian children and adolescents of both sexes with an attractive face. These children are among those used for cinema and television, advertising, and fashion industry [30,31]. Attractive adult males and females were measured during 
several national beauty competitions, which took place in Italy between 2006 and 2008. They were those admitted to the semi-final and final stages of beauty competitions, and were measured just before the semi-final stage of the relevant competition [32].

\section{Results and Discussion}

\subsection{Total, Midline and Lateral Facial Asymmetry}

In both sexes, attractive and control subjects significantly differed in several of the analyzed symmetry descriptors, which assessed separately the contribution of the midplane $\left(\mathrm{DFS}_{\mathrm{m}}\right)$ and lateral $\left(\mathrm{DFS}_{\mathrm{l}}\right)$ asymmetries to the total facial asymmetry $\left(\mathrm{DFS}_{\mathrm{t}}\right)$ (Table 1$)$. In both sexes, the factor age (two-way factorial analysis of variance) was always significant $(\mathrm{p}<0.001)$, with a general trend of reduced asymmetry with growth and development (Figures 2-4). Similar patterns were obtained by both the lateral symmetry and the midline symmetry. Indeed, as suggested by Klingenberg et al. [37], the analysis of object symmetry (a structure symmetric in itself, because it is intersected by the midsagittal plane) should consider the variations of structural features in the median plane separately from those of the features located in the right and left sides.

Table 1. Two-way factorial analysis of variance. NS: not significant ( $p>0.05)$.

\begin{tabular}{|c|c|c|c|c|c|c|c|c|c|c|c|c|}
\hline & DFSI & DFSm & DFSt & $\%$ & Ft-Ft & Os-Os & En-En & Zy-Zy & T-T & Ala-Ala & Ch-Ch & Go-Go \\
\hline \multicolumn{13}{|l|}{ Females } \\
\hline Age & $<0.0001$ & $<0.0001$ & $<0.0001$ & $<0.0001$ & $<0.0001$ & $<0.0001$ & $<0.0001$ & $<0.0001$ & $<0.0001$ & $<0.0001$ & $<0.0001$ & $<0.0001$ \\
\hline Attractiveness & 0.0001 & $<0.0001$ & $<0.0001$ & $<0.0001$ & NS & 0.0025 & 0.0001 & 0.0040 & NS & $<0.0001$ & 0.0026 & NS \\
\hline Interaction & 0.0299 & $<0.0001$ & NS & NS & $<0.0001$ & $<0.0001$ & NS & $<0.0001$ & $<0.0001$ & $<0.0001$ & $<0.0001$ & 0.0008 \\
\hline \multicolumn{13}{|l|}{ Males } \\
\hline Age & $<0.0001$ & 0.0003 & $<0.0001$ & $<0.0001$ & $<0.0001$ & 0.0003 & $<0.0001$ & $<0.0001$ & $<0.0001$ & $<0.0001$ & $<0.0001$ & $<0.0001$ \\
\hline Attractiveness & 0.0002 & $<0.0001$ & 0.0006 & 0.0009 & 0.0450 & NS & $<0.0001$ & NS & 0.0006 & 0.0004 & 0.0298 & $<0.0001$ \\
\hline Interaction & $<0.0001$ & 0.0438 & $<0.0001$ & $<0.0001$ & $<0.0001$ & NS & $<0.0001$ & $<0.0001$ & $<0.0001$ & NS & NS & 0.0012 \\
\hline
\end{tabular}

According to Haraguchi et al. [17], the level of two-dimensional soft-tissue facial asymmetry was independent from age or sex of the subject. Similar results were reported by Rossi et al. [7] for threedimensional skeletal measurements. In contrast, in healthy subjects Ferrario et al. [3] reported that three-dimensional soft-tissue facial asymmetry decreased from adolescence to adult age, a finding well replicated in the current study for both control and attractive subjects (Figures 2-4). It appears that the level of asymmetry, and its distribution in the population, depends on both the analyzed structures (hard vs. soft tissues), and the used method (two- vs. three-dimensional assessment).

In control male subjects, lateral asymmetry was always larger than midplane symmetry, contributing between $71 \%$ (4-5 year-old boys and 15-17 year-old adolescents) and 91\% (8-10 year-old boys) to the total facial asymmetry. In attractive boys and men, a different pattern was observed, with lateral asymmetry explaining only up to 68\% (4-5 year-old boys) of total asymmetry.

Similar patterns of lateral/ midplane asymmetry contributions were observed in control (between 46 and 96\%) and attractive (between 49 and 61\%) females. For control subjects, these values were 
somewhat lower than those previously reported by Ferrario et al. [3] for healthy adolescents and young adults.

Figure 2. Midplane $\left(\mathrm{DFS}_{\mathrm{m}}\right)$ and lateral $\left(\mathrm{DFS}_{\mathrm{l}}\right)$ soft-tissue facial asymmetry in attractive (A) and control (C) females.

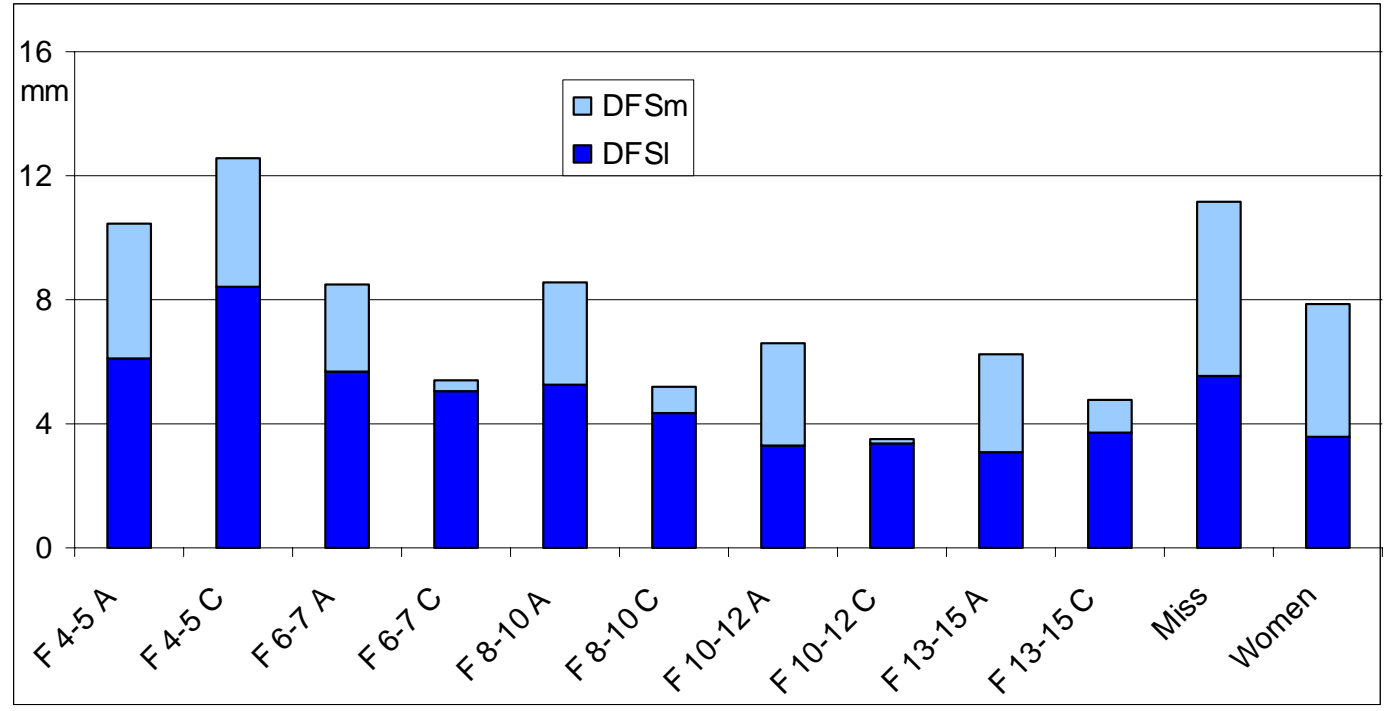

Figure 3. Midplane $\left(\mathrm{DFS}_{\mathrm{m}}\right)$ and lateral $\left(\mathrm{DFS}_{\mathrm{l}}\right)$ soft-tissue facial asymmetry in attractive (A) and control (C) males.

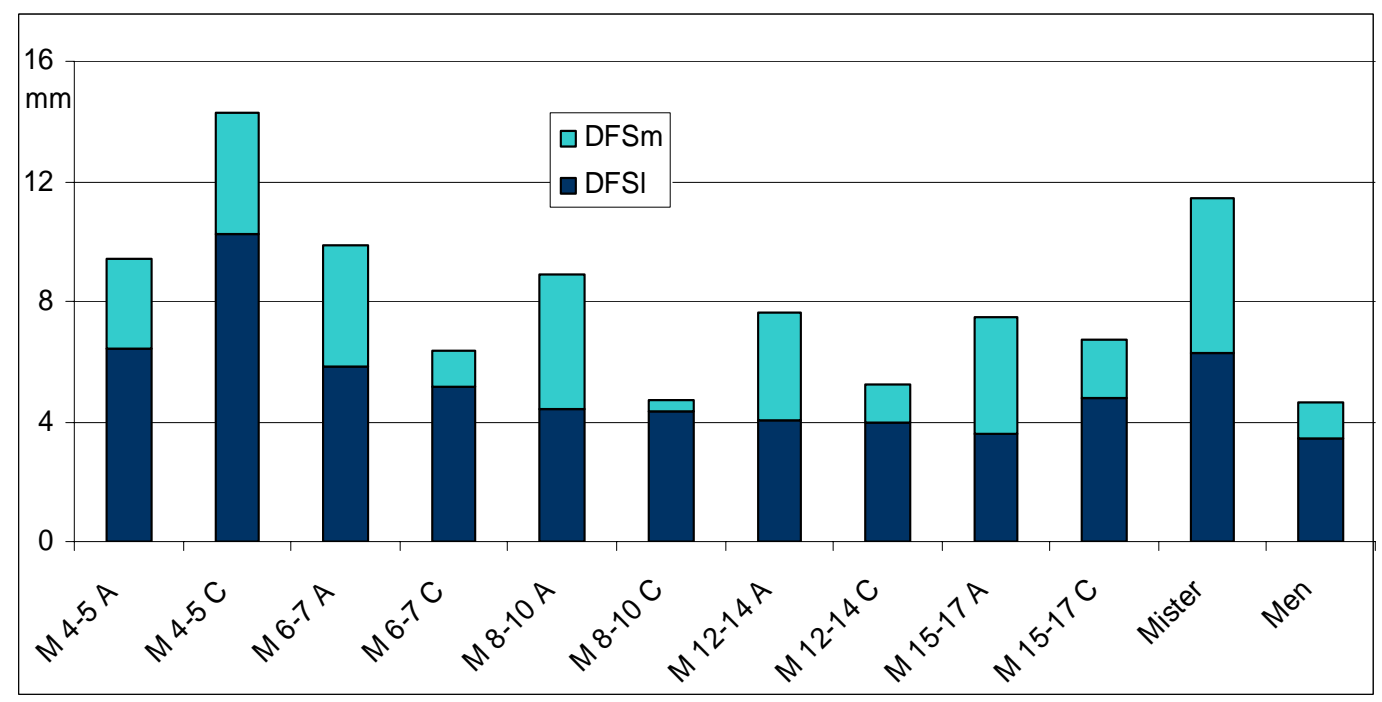

Percentage asymmetry was assessed to account for the different facial dimensions in the analyzed age groups and in the two sexes. In the youngest girls, percentage asymmetry was on average $20-25 \%$ of the nasion-facial barycenter distance, and it reduced to $15 \%$ in the subsequent age groups (Figure 4). In adolescent girls and in adult women, it remained lower than 12\%, except in attractive women ("Miss"), with an average percentage asymmetry of about 15\%. For control "old" adolescent girls and women, these values were very similar to those previously reported in literature [3]. 
Figure 4. Percentage (\%) soft-tissue facial asymmetry in attractive and control persons as a function of sex (F, female; $\mathrm{M}$, male) and age.

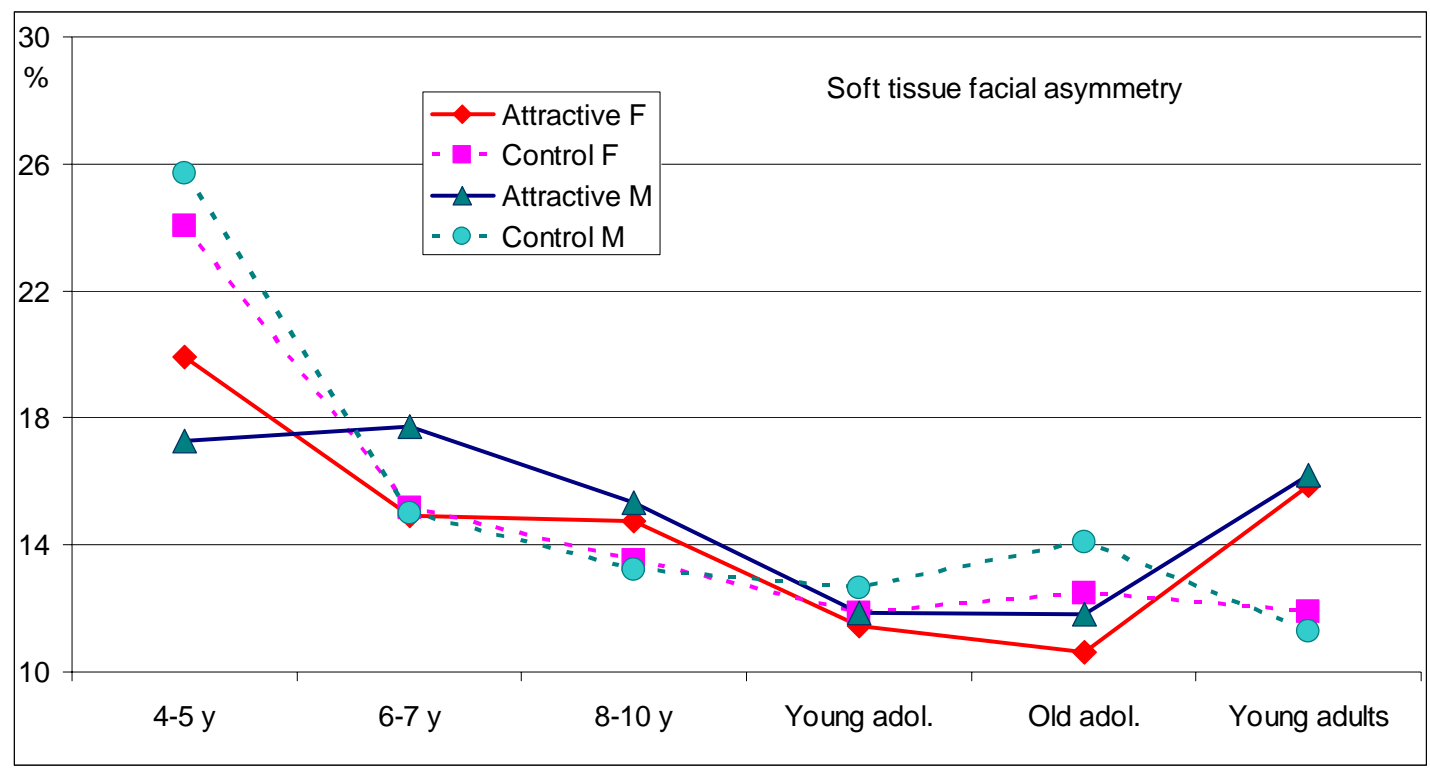

Similar values were found in boys: the average percentage asymmetry was between 20 and $25 \%$ in the youngest age group, with subsequent reductions of around 15\%. Adolescent and adult males had average percentage asymmetry values very close to those found in females (around 12\%); the largest value was found in the attractive men ("Mister"). Literature reports for adolescent boys aged 12 to 15 years (comparable with the current "young" adolescent boys) and for young adult men are in good accord with the current values [3].

Within sex, lateral face, midline face, and total asymmetry (both as an absolute value and as a percentage of facial anteroposterior dimension) were significantly different between attractive and control persons (factor group, analysis of variance, $\mathrm{p}<0.001$, Table 1 ). In almost all cases, there was a significant group $\mathrm{x}$ age interaction (analysis of variance, $\mathrm{p}<0.05$ ), that is the group (attractive vs. control subjects) with the largest asymmetry varied as a function of age. Lateral facial asymmetry, total asymmetry, and percentage asymmetry were both larger (4 to 5 year-old boys and girls) and smaller (adult males and females) in normal versus attractive persons (Student's t tests, $\mathrm{p}<0.003$, corrected for multiple testing). Midline asymmetry was larger in attractive (6 to 7 year-old and 8 to 10 year-old girls and boys; young adolescent girls and boys; old adolescent girls; adult persons; Student's t tests, $\mathrm{p}<0.003$, corrected for multiple testing) than in control subjects.

\subsection{Landmark Asymmetry}

Soft-tissue paired asymmetry increased from the upper to the middle and lower parts of the face (in all groups, gonion was more asymmetric than frontotemporale or orbitale superior, Figures 5-8), as reported in previous investigations for both hard and soft tissues [1,3,5,14,17]. An increment of asymmetry was also observed for the most lateral landmarks when compared to those nearer to the midline, as previously reported [1,5,14]. Landmark asymmetry significantly reduced as a function of age, in accord with some [3], but not all $[7,17]$ previous reports. 
Attractiveness significantly influenced the asymmetry of most of the analyzed paired landmarks, with several group $\mathrm{x}$ age interactions (analysis of variance, Table 1). In females, the frontotemporale landmark was more asymmetric in the 4 to 5 year-old control girls than in the attractive girls of the same age group ( $\mathrm{p}<0.001$, Student's t test, Figure 5). In contrast, this landmark was somewhat more asymmetric in attractive than in control women, but the difference did not reach statistical significance (Figure 6). A similar pattern was found for zygion, with significant differences for both 4 to 5 year-old girls (control more asymmetric than attractive girls), and adult women (attractive more asymmetric than control women).

Figure 5. Soft-tissue facial landmark asymmetry in attractive (A, continuous lines, closed symbols) and control (C, interrupted lines, open symbols) girls.

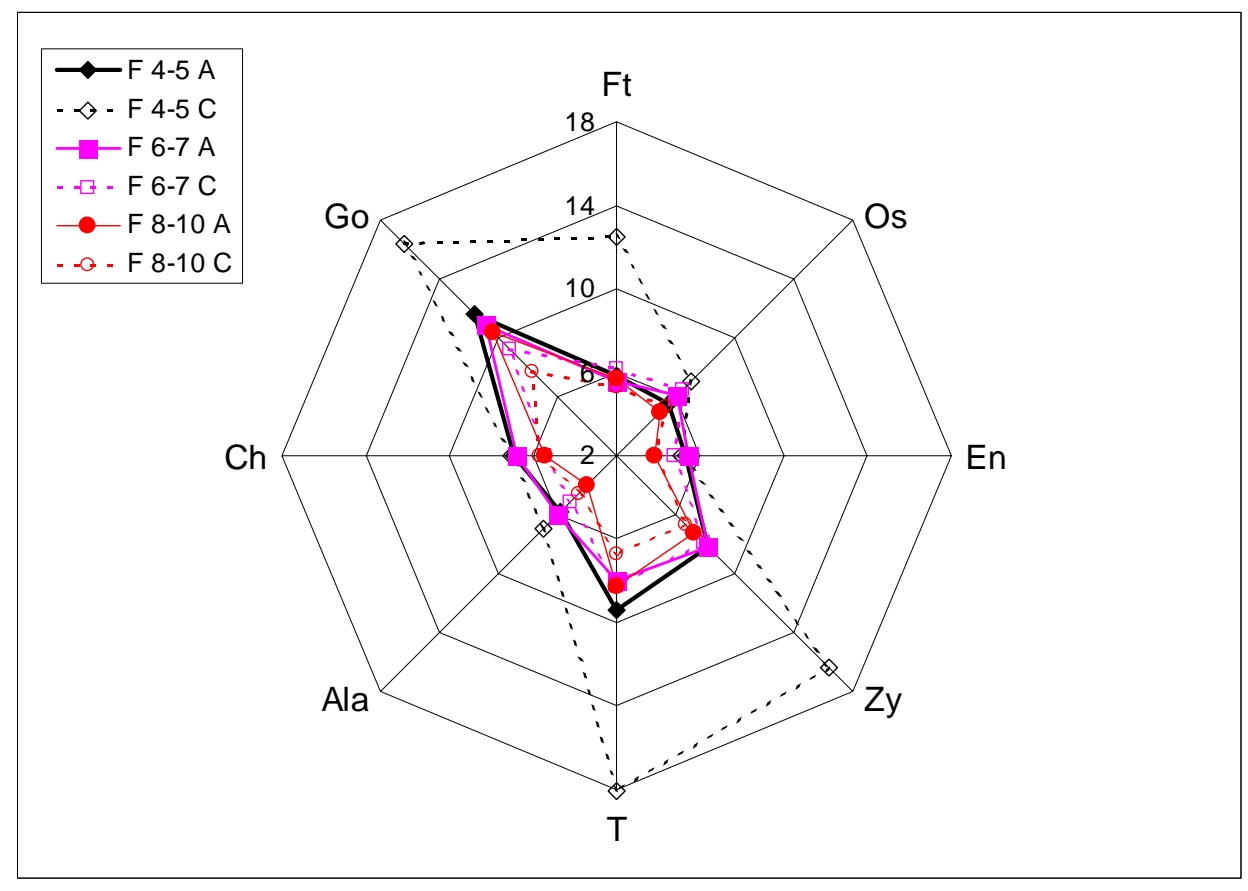

A significant effect of attractiveness was found for both eye landmarks (orbitale superior and endocanthion); orbitale superior was somewhat more asymmetric in control than in attractive girls, but the difference reversed in the oldest age classes. On no occasion, did it reach statistical significance ( $p>0.003$, post hoc tests). Endocanthion was always more asymmetric in attractive than in control females; the post hoc test was significant for adult women $(\mathrm{p}<0.001)$. In the upper and middle parts of the face, mean values of landmark asymmetry were well comparable to those reported by Shaner et al. [14].

Tragus and gonion asymmetries were not significantly influenced by attractiveness (analysis of variance, $\mathrm{p}>0.05$ ), but significant age $\mathrm{x}$ group interactions were found: for both landmarks, asymmetry was significantly larger in control 4 to 5 year-old girls, and in attractive women, than in their coetaneous peers. In the facial middle and lower thirds, ala nasi and cheilion were somewhat more asymmetric in control young female persons than in attractive persons of the same age; in the adult group, attractive females were significantly more asymmetric in the nose and mouth than control ones. 
Figure 6. Soft-tissue facial landmark asymmetry in attractive (A, continuous lines, closed symbols) and control (C, interrupted lines, open symbols) adolescent girls and women.

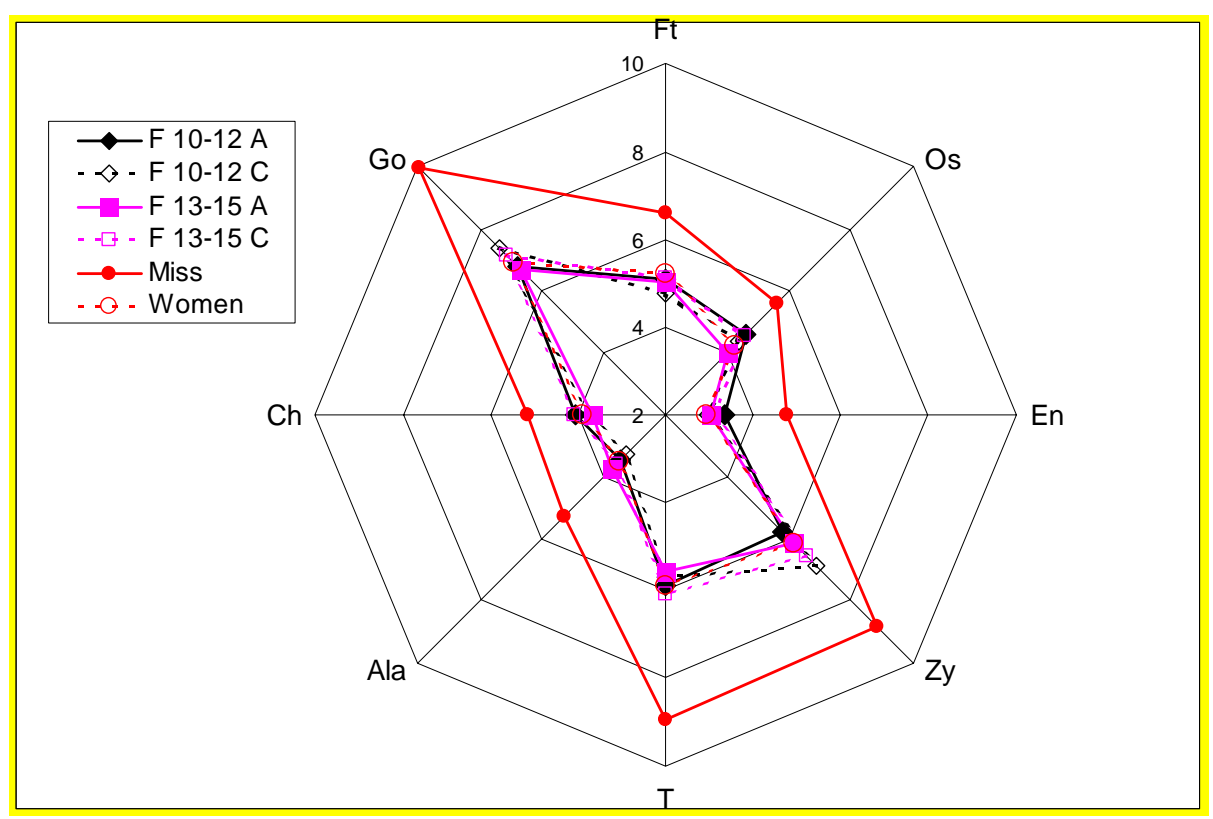

Similar patterns were found in the male group: Landmark asymmetry was relatively reduced in attractive boys, and relatively increased in attractive men, when compared to their age-related control persons. The frontotemporale and zygion landmarks were more asymmetric in the 4 to 5 year-old control boys than in the attractive boys of the same age group ( $<0.001$, Student's $t$ test, Figure 7 ), but more asymmetric in attractive than in control men ("Mister", $\mathrm{p}=0.003$, Figure 8).

Figure 7. Soft-tissue facial landmark asymmetry in attractive (A, continuous lines, closed symbols) and control (C, interrupted lines, open symbols) boys.

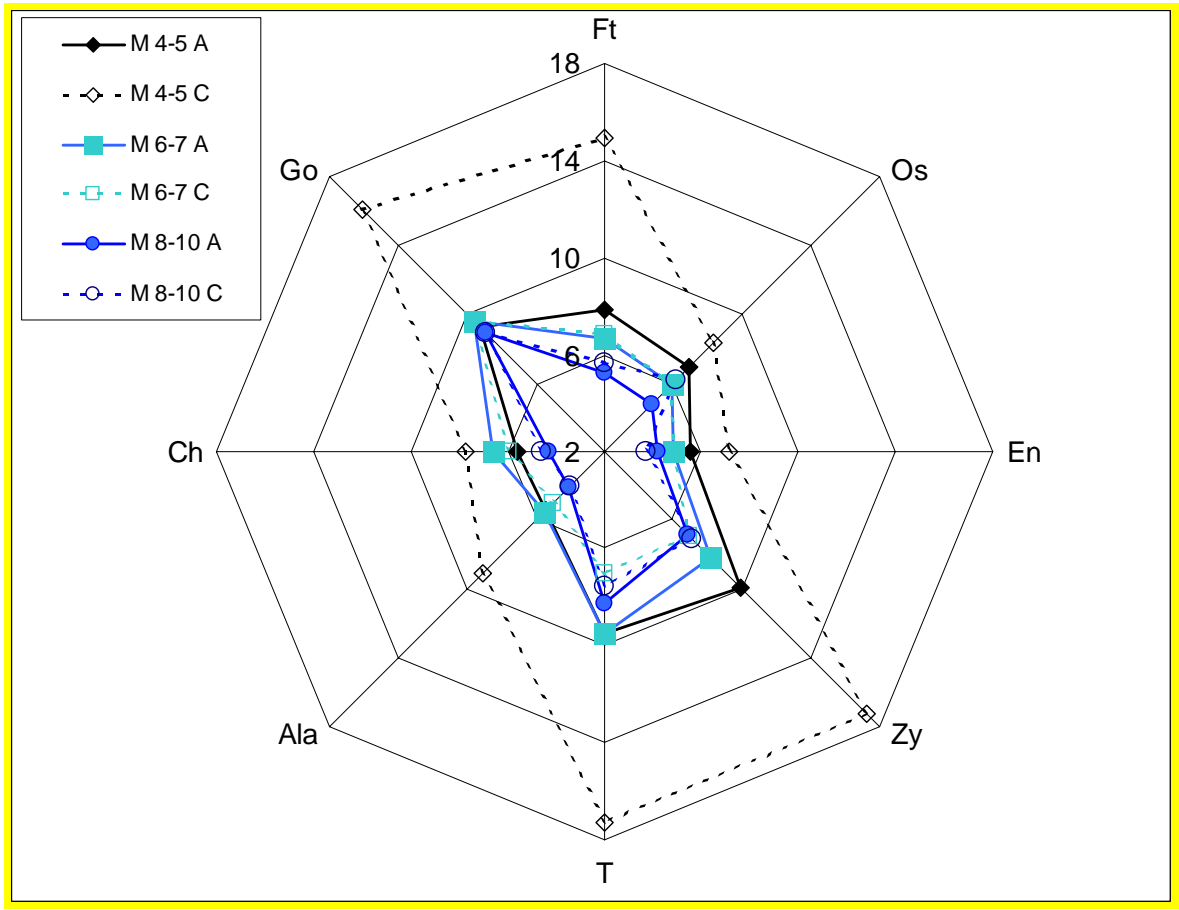


Figure 8. Soft-tissue facial landmark asymmetry in attractive (A, continuous lines, closed symbols) and control (C, interrupted lines, open symbols) adolescent boys and men.

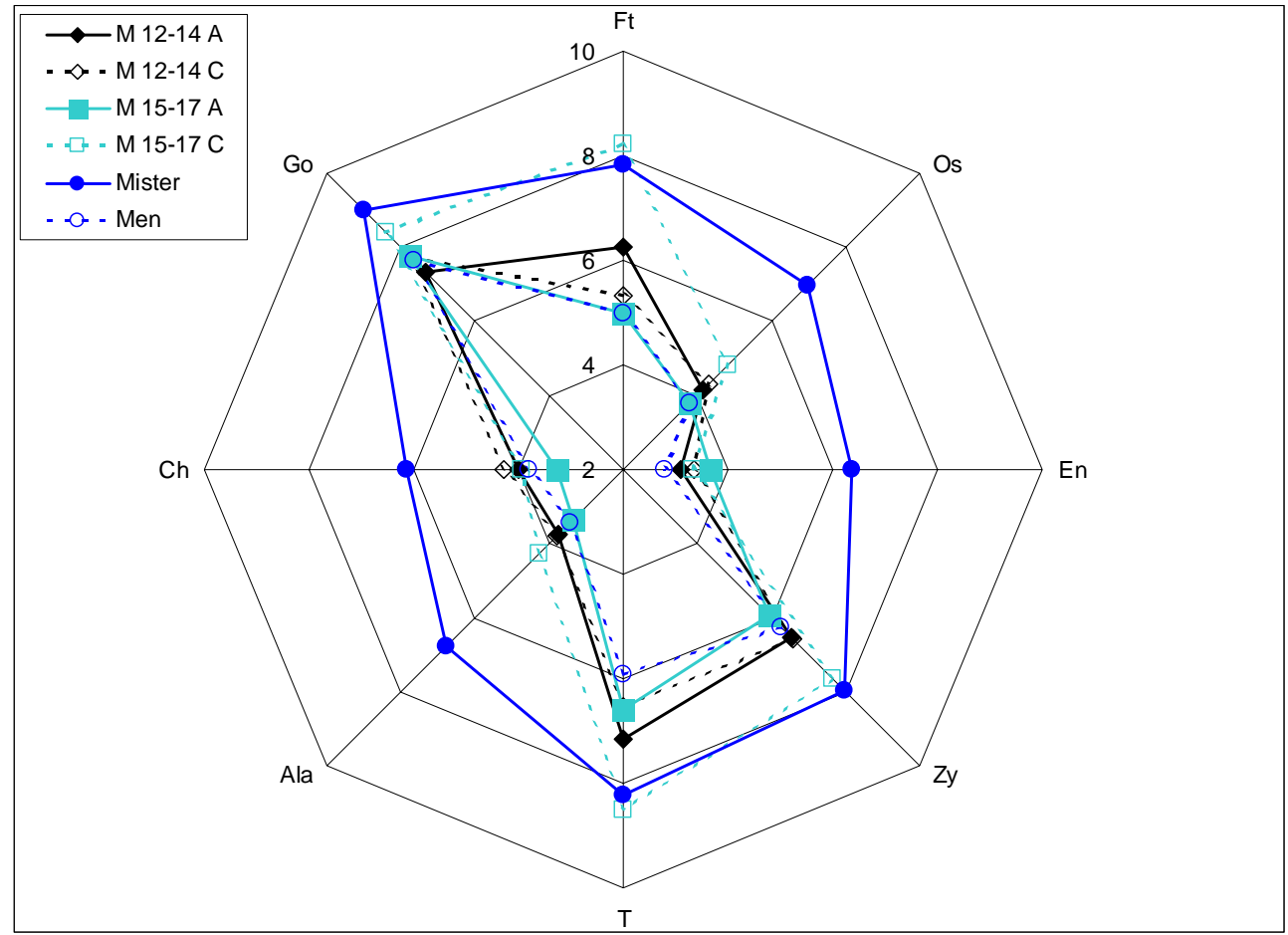

Significant effects of attractiveness, and significant age $\mathrm{x}$ group interactions, were found for endocanthion, tragus and gonion; all these landmarks were more asymmetric in control than in attractive boys, but the difference reversed in the oldest age classes. For endocanthion and tragus, the post hoc tests were significant for adult men $(\mathrm{p}<0.001)$; for tragus and gonion, they were significant for 4 to 5 year-old boys ( $\mathrm{p}=0.002$ ). Also, ala nasi and cheilion were somewhat more asymmetric in control than in attractive boys; in the adult group, attractive males had a significantly more asymmetric nose and mouth than control ones $(\mathrm{p}<0.001)$.

For adolescent boys and girls, and for young adults, the current mean values of landmark asymmetry were comparable to those previously reported by Ferrario et al. [3].

Overall, in the males and females analyzed in the current study, the effect of attractiveness on facial asymmetry (both total, and divided into paired landmarks) depended on age: young attractive persons were less asymmetric than control persons of the same sex and age, but in adult persons an opposite pattern was found. Considering the age effect, it is difficult to isolate single landmarks as the most effective in determining facial asymmetry in the two analyzed groups of subjects. Indeed, this separation is better done when control subjects are compared to patients with a clear facial asymmetry, such as those with unilateral cleft lip/palate $[8,39,40]$.

This age-related pattern of asymmetry/ symmetry versus attractiveness has never been reported in previous investigations, because the impact of asymmetry on facial attractiveness had previously only been analyzed in adult persons. Further studies should be performed for children and adolescents. Obviously, the selection of soft-tissue landmarks used may play a role in the quantification of asymmetry: All landmarks had been selected in previous studies made on normal persons [3], and were in good accord with those suggested by Little et al. [24] for the analysis of facial attractiveness. 
Even if the male-female differences in facial asymmetry, and its relationship with attractiveness, was not the actual topic of the current investigation, and no formal statistical tests were made to compare male versus female values, some sex-related differences were noted in the pattern of landmark asymmetries. For instance, in females the asymmetry of orbitale superior landmark was significantly influenced by attractiveness, while that of frontotemporale landmark was not (Table 1). This pattern reversed in male subjects, who had significant differences in frontotemporale, but not in orbitale superior. Considering that no previous study measured the actual influence of single landmarks on facial attractiveness in the two sexes, the significance of this different pattern is not known; nonetheless, both landmarks describe the lateral and upper parts of the face (Figure 1).

Zygion and cheilion landmark asymmetries were significantly influenced by attractiveness in females but not in males: The importance of relatively prominent cheekbones, and full and prominent lips for female attractiveness should be taken into consideration [20,21,28-32]. Additionally, Ercan et al. [1] found that the landmark zygion was the endpoint of the larger number of asymmetric facial distances, and the effect was more evident in adult females than in males. In the current study, within each age and study group (control and attractive persons), the mean values of asymmetry were similar in males and females, in contrast with Ercan et al. [1] who found a larger asymmetry in females than in males.

Tragus and gonion landmark asymmetries were significantly influenced by attractiveness in males but not in females: Male attractiveness has been reported to be positively influenced by facial markers of high testosterone levels, with a more developed facial lower third [20,22,25,30,33].

\subsection{General Discussion}

Subtle differences between the two facial halves are common findings even in healthy persons [1-9], the origin of such is still being discussed. Both epigenetic (environmental) and genetic (mostly explained by epistatic interactions of genes) factors have been recognized [4]. According to the theories that assign the major role to environmental factors, individuals with the best buffering capacities possess the least fluctuating asymmetry [4], and may be more attractive: Their pleasant aspect should be a signal of better health and mate quality [13,19,21-24,27]. Despite this framework, all previous investigations that assessed independently selected attractive persons [5,18] found a significant facial asymmetry in both the skeletal and cutaneous structures, in good accord with the current findings. As reported by Springer et al. [27], mild asymmetries do not alter a generally attractive face, and the effect becomes smaller as more lateral the asymmetric trait is. This concords with the higher levels of fluctuating asymmetry found for the lateral facial landmarks (tragus, gonion, zygion) when compared with those nearer to the midline (endocanthion, orbitale superius, nasal alar crest, cheilion), as shown in Figures 5-8. The increased level of asymmetry in facial lateral structures, found both in control subjects and in attractive persons, concords with previous reports [1,5,14]. Midline and dental asymmetries are always perceived as unattractive [41].

The asymmetries in facial function (larger movements on the left than on the right side of the face) [10-12], as well as the most recent reports on the strict relationship between full and hemi-face attractiveness [36], are explained by asymmetries in human cerebral hemispheres, with resulting asymmetries in visual perception and interpretation of the surrounding world: The left side of the face 
receives a larger attention from the viewer [10,12,17,19,36,38]. Considering the importance of expressive features in the assessment of facial attractiveness [21], our asymmetric brain perception may explain the importance of mild facial asymmetries [36]. Additionally, the different nerve supplies of the upper (bilateral central nervous system commands to the facial nerve nuclei) and lower (only commands to the contralateral nucleus) parts of the face may explain the special asymmetry in mouth and lip movements [1,36].

The current results, therefore, contrast with the view that, in adult males and females, a reduced fluctuating asymmetry is constantly associated with increased facial and body attractiveness [13,19,21-24,27]: This finding may apply to children and adolescents, but not completely to young adults, at least when attractive persons were chosen by professionals of the media world. Indeed, in the current study, attractive persons were selected by professionals of the television/cinema/advertisement (juries of beauty competitions, casting agency experts), and not by lay people [41]. The influence of the different kinds of "experts" has been assessed in previous studies, but with contrasting results: In the assessment of facial esthetics, the specialized opinions of dental and surgical professionals had been found to be relatively more critical $[41,42]$, while those of plastic surgeons have been reported to be less critical than those of non-professionals [43]. On no occasion has the opinion of specialists from the media world been compared to that of other groups, but it may play a role in the definition of the criteria of facial attractiveness. Therefore, in the current group of young adults, the perception of attractiveness for all finalists or semi-finalists of national beauty competitions, is probably accredited to cues other than symmetry, as already shown: neoteny, youthfulness, and sexual dimorphism for women [32]; sexual dimorphism for men [31]. Obviously, none of the analyzed persons had largely asymmetric traits, and all the asymmetry indices were minor when compared to values collected in patients with cleft lip and palate [39].

Indeed, other investigators reported that facial symmetry did not have a significant effect on the perception of attractiveness $[5,12,18,36,38]$. The lack of an increased soft tissue facial asymmetry in patients with genetic syndromes also diverges with a strict relationship between symmetry, health, genetic stability and attractiveness [14]. In men, Penton-Voak et al. [19] reported that facial symmetry was not solely an explanation for attractiveness. Apparently, facial symmetry plays a larger role in mate choice than for attractiveness judgment in general [23]. In women, facial symmetry, sexual dimorphism and averageness co-vary, and their effects on facial attractiveness cannot be easily separated [21,22,24]. In particular, asymmetric faces depart from attractiveness because they deviate from averageness [21]. Also, the importance of single traits (eyes, mouth, nose) to facial attractiveness should be taken into consideration, especially for women [21,22,27,32]. In synthesis, humans' facial symmetry may be a good sign of health, but not necessarily for attractiveness, also considering the intrinsic asymmetry of our brain, and, therefore, of our perceptions [36,38].

The method used to investigate the relationship between facial attractiveness and asymmetry seems to play a significant role: Whenever stimuli are obtained by digital morphing and averaging, a positive correlation is found $[25,26]$. In contrast, when computer constructed left-left or right-right faces are used, attractiveness is not explained by bilateral symmetry. A recent investigation compared the attractiveness ratings of both full-faces (frontal view) and hemi-faces (only right or left side of the face), finding a strong correlation between them [36]. The authors concluded that in humans, bilateral facial symmetry is not a major factor in beauty assessment. 


\subsection{Study Limitations}

Among the study limitations there is the method used for landmark digitization. Data collection was made with contact instruments, that digitize only single, selected landmarks neglecting the surface comprised between them $[40,44]$. These instruments necessitate a relatively long data collection time (approximately $60 \mathrm{~s}$ for a set of 50 landmarks, which is currently considered a good compromise between digitization time and accuracy of facial description), which is more time consuming than that needed with optical instruments (10-60 ms, depending on the instrument), with possible movement artifacts; nevertheless, the carefully controlled protocol limited this problem [3,30-32].

Additionally, optical scanners provide complete information about facial surface characteristics, and are currently being used both in normal persons and in patients before and after orthopedic, orthodontic and surgical treatments [6,9,34,40,45-47]. The current method neglected all surface information. Nonetheless, optical scanners are often used only for data collection, and all calculations are subsequently performed on sets of facial landmarks, with a procedure similar to that used for the current investigation [14,34,40]. Biologically meaningful landmarks are the basis of face recognition and analysis [48], and were also previously used for symmetry analysis [1,5,14]. Recent investigations assessed the performance of contact instruments in clinical practice, and found their usage satisfactory, also considering that these instruments can be used directly in any environment, for instance quickly meeting the attractive subjects during beauty competitions, fashion events, or castings [30-31,49]. In contrast, optical instruments (laser scans, stereophotogrammetric digitizers) usually need dedicated locations, which often cannot be organized outside specialized laboratories.

In the current preliminary study, we assessed single landmarks (for those on the midsagittal plane) and pairs of landmarks (for the paired ones) using univariate methods that were developed in our laboratory to investigate the effects of sex, age, pathology and relevant treatments within biological and medical contexts $[3,8,39]$. Further investigations may take advantage of more comprehensive methods of analysis, such as those provided by geometric morphometrics and shape analysis $[1,6,13,15,34,37,40,48,50]$.

Another study limitation is its cross-sectional nature; different persons were examined in different age classes. Furthermore, some age classes were less numerous than others, and further data collections should be planned specifically for children [30].

\section{Experimental Section}

\subsection{Analyzed Subjects}

One thousand and twenty nine white Caucasian, northern Italian persons aged between four and 30 years were analyzed. Six hundred and sixty nine (397 males, 272 females) were reference subjects; all were healthy, with normal dentofacial dimensions and proportions; no subjects had a previous history of craniofacial surgery, trauma or congenital anomalies. Three hundred and eighty (148 males, 232 females) were attractive subjects, selected either by casting agencies (children and adolescents, 4 to 17 years of age) or by juries of national beauty competitions (adult men and women, 18 to 30 years of age). Selected facial linear distances, angles, volumes and areas collected from both attractive and control persons were previously published [30-32], but on no occasion was data about facial 
symmetry published. All the analyzed persons, and the parents/ legal guardians of those under 18 years of age, gave their informed consent to the experiment. All procedures were not invasive, did not provoke damage, risks or discomfort to the subjects, and were preventively approved by the local ethic committee.

\subsection{Collection of Facial Landmarks}

A detailed description of the data collection procedure can be found elsewhere $[3,30-32,44,51]$. In summary, the procedure takes place in two separate steps, and is followed by off-line calculations. At first, for each person, a set of 50 soft-tissue landmarks were located and marked on the cutaneous surface using a black eyeliner (Figure 1). The three-dimensional (x, y, z) coordinates of the facial landmarks were obtained with three-dimensional computerized digitizers (an electromagnetic threedimensional tablet, and an electromechanical instrument). Data collection was performed with the subject sitting in a natural head position in a chair with a backrest. Landmark digitization took approximately one minute for each subject. Files of the three-dimensional coordinates were obtained, and original computer programs were used for all subsequent off-line calculations.

\subsection{Method Error}

The main sources of error in the method used in the current study were the identification of facial landmarks and their digitization, because subsequent calculations were done automatically by computerized algorithms [3,8,39,51]. Correct identification and marking of the 50 facial landmarks was checked before data collection, and any incorrect mark was cleaned and repositioned [44].

Digitization errors can occur due to technical errors in the instrument (checked by repeated measurements of geometrical objects of known dimensions), movement artifacts, and operator errors. Both systematic and random errors were assessed using the stone cast of a male face, and comparing the measurements obtained by the computerized digitizer with those obtained by standard calipers. For systematic errors, the mean difference between pairs of measurements was $0.22 \mathrm{~mm}$ (SD 0.7), which did not differ significantly from the expected value of 0 . For random errors, we found that the coefficients of variation of repeated measurements ranged between $0.28 \%$ and $1.10 \%$ (mean $0.54 \%$ ), and all intra-class correlation coefficients (ICC) were larger than $0.999[8,51]$.

Major movement artifacts were immediately checked with on-line computerized facial reconstructions: Whereas the facial morphology was grossly altered, the landmark coordinates were digitized again. The effect of minor movement artifacts was assessed by repeated identification and digitization of the 50 landmarks directly on the subject faces. No systematic errors were found (paired Student's t test, $\mathrm{p}>0.01$ ). Random variations (Technical Error of Measurement, TEM, or Dahlberg's error) for all 50 landmarks were $1.20 \mathrm{~mm}$ in males (1.04\% of the nasion-mid tragion distance), and $0.95 \mathrm{~mm}$ in females (1.05\% of the nasion-mid tragion distance) [51]; for selected distances, TEMs ranged between 0.25 and 0.32 , and the ICC were between 0.66 and 0.69 [8]. Overall, in vivo error seemed to influence landmark coordinates by about 1\% [8], well above the total percentage asymmetry found in the current groups of persons (Figure 4). 


\subsection{Calculation of Facial Asymmetry}

In each subject, facial asymmetry was assessed as detailed by Ferrario et al. [3]. The procedure involved calculation of: a) The facial plane of symmetry in the three-dimensional space, computed as the vertical plane passing through the nasion landmark and perpendicular to the plane connecting the two exocanthi); b) the barycenters (BC) of the right- and left-side facial halves (using the $\mathrm{x}, \mathrm{y}, \mathrm{z}$ coordinates of the landmarks endocanthion, orbitale superius, frontotemporale, zygion, tragion, nasal alar crest, cheilion and gonion); the $\mathrm{BC}$ of the right-side part of the face was mirrored on the left-side part of the face by inverting the sign of its $x$ (right-left) coordinate [46].

The three-dimensional distance between the left-side BC and the mirrored right-side BC, provided a facial lateral asymmetry (DFS; ; unit, mm). This will be zero if the arrangement of the analyzed paired landmarks is equal on the right and left sides of the face $[37,46]$.

The deviation of midline landmarks nasion, pronasale, subnasale, pogonion and menton from the plane of symmetry provided a facial midline asymmetry (distance from the symmetry, $\mathrm{DFS}_{\mathrm{m}}$; unit, $\mathrm{mm}$ ); if all landmarks are exactly on the midline, a 0 asymmetry will result. A total asymmetry was then obtained as the sum of the lateral and midline asymmetries $\left(\mathrm{DFS}_{\mathrm{t}}=\mathrm{DFS}_{\mathrm{l}}+\mathrm{DFS}_{\mathrm{m}}\right.$; unit, mm). To obtain a single value independent of facial dimensions, total facial asymmetry was also expressed as a percentage (DFS\%) of the nasion-BC distance (average between the left and right side), a global index of facial size.

Localized facial asymmetry was also obtained considering the separate contributions of the paired facial landmarks (Ft, Os, En, Zy, T, Ala, Ch, and Go) to the lateral facial asymmetry DFS .

\subsection{Statistical Analysis}

Attractive and normal persons were divided into several age and sex groups; for children and adolescents, age groups extended over two-three years each, while adult persons were analyzed in a single group (Table 2). Descriptive statistics (mean and standard deviation) for $\mathrm{DFS}_{\mathrm{t}}, \mathrm{DFS}_{\mathrm{l}}, \mathrm{DFS}_{\mathrm{m}}$, DFS\%, and for the asymmetry of paired facial landmarks were computed within group (attractive and normal persons), sex (males, females), and age group (children: 4-5 years, 6-7 years, 8-10 years; adolescent girls: 10-12 years, 13-15 years; adolescent boys: 12-14 years, 15-17 years; young adult women; young adult men). These values described the presence of mean asymmetric facial features in the individual subjects. For adolescent subjects, different age groups were made for "young” and "old" boys and girls because of the age-related differences in pubertal growth [31].

Within sex, mean values between attractive and control subjects (factor 1, two levels), and age groups (factor 2, six levels) were compared using a two-way factorial analyses of variance. The group $\mathrm{x}$ age interaction was also computed. Post-hoc Student's t tests were made when appropriate. Significance was set at 5\% ( $\mathrm{P}<0.05$ for global analysis; $\mathrm{P}<0.003$ for post hoc tests, after Bonferroni correction for multiple testing), with two-tail statistical tests used in all analyses. 
Table 2. Analyzed subjects in the various age and sex groups.

\begin{tabular}{|c|c|c|c|c|c|c|}
\hline & \multicolumn{3}{|c|}{ Children } & \multicolumn{2}{|c|}{ Adolescents } & \multirow{2}{*}{$\begin{array}{c}\text { Adults } \\
18-30 \text { y }\end{array}$} \\
\hline & 4-5 y & $6-7 y$ & $8-10 y$ & "Young"* & “Old"§ & \\
\hline \multicolumn{7}{|l|}{ Females } \\
\hline Attractive & 14 & 18 & 14 & 24 & 23 & 139 \\
\hline Control & 11 & 42 & 42 & 48 & 60 & 69 \\
\hline \multicolumn{7}{|l|}{ Males } \\
\hline Attractive & 8 & 21 & 13 & 21 & 24 & 60 \\
\hline Control & 19 & 41 & 55 & 99 & 55 & 128 \\
\hline
\end{tabular}

\section{Conclusions}

In the current study, attractive children, adolescents and young adults of both sexes were selected by professionals of the media world, and the soft tissue characteristics of their faces were compared to those collected in control subjects of the same age, sex and ethnic group. Soft-tissue facial asymmetries reduced as a function of age in both males and females. Attractive children were more symmetric than control children, but the reverse was true of young adults. The effect of symmetry on attractiveness seemed to change as a function of age. Soft-tissue paired asymmetry increased from the upper to the middle and lower parts of the face (in all groups, mandibular asymmetry was larger than orbital asymmetry). Some sex-related differences were noted in the pattern of landmark asymmetries: Cheek and labial asymmetries were significantly influenced by attractiveness in females but not in males, while mandibular asymmetries were significantly influenced by attractiveness in males but not in females. It seems that those structures that are most influential for facial attractiveness in the two sexes are also those most affected by facial asymmetry.

One of the differences between the current and most of previous studies, on the effect of various facial traits (symmetry, averageness, sexual dimorphism) on facial attractiveness, is that we measured existent persons, and tried to find measurable specific characteristics. We did not rely on subjective judgments, but only on objective data. Rhodes et al. [26] stated that subjective judgment is better than objective measurement, because single values cannot encompass all facial details, which are better appreciated by the acute sensitivity of the human visual system. From this point of view, the current three-dimensional assessment of facial asymmetry took almost the whole face into consideration, and was not limited to single left-right discrepancies [3,21]. Nonetheless, more refined mathematical and geometrical methods are necessary to better appreciate the intermingled effects of symmetry and attractiveness in all ages and in both sexes [1,6,13,34,37,40,48,50].

\section{Acknowledgements}

The authors thank all the Staff of the LAFAS, Università degli Studi di Milano, who participated in data collection and analysis ( Miriam Binelli, Francesca Catti, Veronica Ciusa, Anna Colombo, Iolanda Decorato, Claudia Dellavia, Francesca R. Dimaggio, Silvia Florean, Fabrizio Mian, Matteo Rossini, Gianluca M. Tartaglia, Davide G. Tommasi). Patrizia Frangella organized the data collection in 
attractive persons. The expert secretarial assistance of Cinzia Lozio is gratefully acknowledged. Financial support was obtained from the Università degli Studi di Milano, and from the Board of Directors of the Società Italiana Di Ortodonzia (SIDO).

\section{References}

1. Ercan, I.; Ozdemir, S.T.; Etoz, A.; Sigirli, D.; Tubbs, R.S.; Loukas, M.; Guney, I. Facial asymmetry in young healthy subjects evaluated by statistical shape analysis. J. Anat. 2008, 213, 663-669.

2. Ferrario, V.F.; Sforza, C.; Schmitz, J.H.; Santoro, F. Three-dimensional facial morphometric assessment of soft tissue changes after orthognathic surgery. Oral Surg. Oral Med. Oral Pathol. Oral Radiol. Endod. 1999, 88, 549-556.

3. Ferrario, V.F.; Sforza, C.; Ciusa, V.; Dellavia, C.; Tartaglia, G.M. The effect of sex and age on facial asymmetry in healthy subjects: a cross-sectional study from adolescence to mid-adulthood. J. Oral Maxillofac. Surg. 2001, 59, 382-328.

4. Leamy, L.J.; Klingenberg, C.P. The genetics and evolution of fluctuating asymmetry. Annu. Rev. Ecol. Evol. Syst. 2005, 36, 1-21.

5. Peck, S.; Peck, L.; Kataja, M. Skeletal asymmetry in esthetically pleasing faces. Angle Orthod. 1991, 61, 43-48.

6. Ramieri, G.A.; Nasi, A.; Dell'acqua, A.; Verzé, L. Facial soft tissue changes after transverse palatal distraction in adult patients. Int. J. Oral Maxillofac. Surg. 2008, 37, 810-818.

7. Rossi, M.; Ribeiro, E.; Smith, R. Craniofacial asymmetry in development: an anatomical study. Angle Orthod. 2003, 73, 381-385.

8. Sforza; C.; Peretta, R.; Grandi, G.; Ferronato, G.; Ferrario. V.F. Three-dimensional facial morphometry in skeletal Class III patients. A non-invasive study of soft-tissue changes before and after orthognathic surgery. Br. J. Oral Maxillofac. Surg. 2007, 45, 138-144.

9 Stauber, I.; Vairaktaris, E.; Holst, A.; Schuster, M.; Hirschfelder, U.; Neukam, F.W.; Nkenke, E. Three-dimensional analysis of facial symmetry in cleft lip and palate patients using optical surface data. J. Orofac. Orthop. 2008, 69, 268-282.

10. Borod, J.C.; Koff, E.; Yecker, S.; Santschi, C.; Schmidt, J.M. Facial asymmetry during emotional expression: gender, valence, and measurement technique. Neuropsychologia 1998, 36, 1209-1215.

11. Okamoto, H.; Haraguchi, S.; Takada, K. Laterality of asymmetry in movements of the corners of the mouth during voluntary smile. Angle Orthod. 2010, 80, 223-239.

12. Zaidel, D.W. In Neuropsychology of Art; Psychology Press: Howe, East Sussex, UK, 2005; Chapter 9, pp. 168-169.

13. Brown, W.M.; Price, M.E.; Kang, J.; Pound, N.; Zhao, Y.; Yu, H. Fluctuating asymmetry and preferences for sex-typical bodily characteristics. Proc. Natl. Acad. Sci. USA 2008, 105, 12938-12943.

14. Shaner, D.J.; Peterson, A.E.; Beattie, O.B.; Bamforth, J.S. Assessment of soft tissue facial asymmetry in medically normal and syndrome-affected individuals by analysis of landmarks and measurements. Am. J. Med. Genet. 2000, 93, 143-154. 
15. Lu, K.H. Harmonic analysis of the human face. Biometrics. 1965, 21, 491-505.

16. Farkas, L.G. Anthropometry of the Head and Face, 2th ed.; Raven Press: New York, NY, USA, 1994.

17. Haraguchi, S.; Iguchi, Y.; Takada, K. Asymmetry of the face in orthodontic patients. Angle Orthod. 2008, 78, 421-426.

18. Ing, E.; Safarpour, A.; Ing, T.; Ing, S. Ocular adnexal asymmetry in models: a magazine photograph analysis. Can. J. Ophthalmol. 2006, 41, 175-182.

19. Penton-Voak, I.S.; Jones, B.C.; Little, A.C.; Baker, S.; Tiddeman, B.; Burt, D.M.; Perrett, D.I. Symmetry, sexual dimorphism in facial proportions and male facial attractiveness. Proc. Biol. Sci. 2001, 268, 1617-1623.

20. Bashour, M. History and current concepts in the analysis of facial attractiveness. Plast. Reconstr. Surg. 2006, 118, 741-756.

21. Baudouin, J.Y.; Tiberghien, G. Symmetry, averageness, and feature size in the facial attractiveness of women. Acta Psychol. (Amst). 2004, 117, 313-332.

22. Jones, B.C.; DeBruine, L.M.; Little, A.C. The role of symmetry in attraction to average faces. Percept. Psychophys. 2007, 69, 1273-1277.

23. Little, A.C.; Burt, D.M.; Penton-Voak, I.S.; Perrett, D.I. Self-perceived attractiveness influences human female preferences for sexual dimorphism and symmetry in male faces. Proc. Biol. Sci. 2001, 268, 39-44.

24. Little, A.C.; Jones, B.C.; Waitt, C.; Tiddeman, B.P.; Feinberg, D.R.; Perrett, D.I.; Apicella, C.L.; Marlowe, F.W. Symmetry is related to sexual dimorphism in faces: data across culture and species. PLoS One 2008, 3, e2106.

25. Rhodes, G. The evolutionary psychology of facial beauty. Annu. Rev. Psychol. 2006, 57, 199-226.

26. Rhodes, G.; Yoshikawa, S.; Palermo, R.; Simmons, L.W.; Peters, M.; Lee, K.; Halberstadt, J.; Crawford, J.R. Perceived health contributes to the attractiveness of facial symmetry, averageness, and sexual dimorphism. Perception 2007, 36, 1244-1252.

27. Springer, I.N.; Wannicke, B.; Warnke, P.H.; Zernial, O.; Wiltfang, J.; Russo, P.A.; Terheyden, H.; Reinhardt, A.; Wolfart, S. Facial attractiveness: visual impact of symmetry increases significantly towards the midline. Ann. Plast. Surg. 2007, 59, 156-162.

28. Matoula, S.; Pancherz, H. Skeletofacial morphology of attractive and nonattractive faces. Angle Orthod. 2006, 76, 204-210.

29. Orsini, M.G.; Huang, G.J.; Kiyak, H.A.; Ramsay, D.S.; Bollen, A.M.; Anderson, N.K.; Giddon, D.B. Methods to evaluate profile preferences for the anteroposterior position of the mandible. Am. J. Orthod. Dentofacial Orthop. 2006, 130, 283-291.

30. Sforza, C.; Laino, A.; D’Alessio, R.; Dellavia, C.; Grandi, G.; Ferrario, V.F. Three-dimensional facial morphometry of attractive children and normal children in the deciduous and early mixed dentition. Angle Orthod. 2007, 77, 1025-1033.

31. Sforza, C.; Laino, A.; D’Alessio, R.; Grandi, G.; Tartaglia, G.M.; Ferrario, V.F. Soft-tissue facial characteristics of attractive and normal adolescent boys and girls. Angle Orthod. 2008, 78, 799-807. 
32. Sforza, C.; Laino, A.; D’Alessio, R.; Grandi, G.; Binelli, M.; Ferrario, V.F. Soft-tissue facial characteristics of attractive Italian women as compared to normal women. Angle Orthod. 2009, 79, 17-23.

33. Fink, B.; Neave, N.; Seydel, H. Male facial appearance signals physical strength to women. Am. $J$. Hum. Biol. 2007, 19, 82-87.

34. Weinberg, S.M.; Naidoo, S.D.; Bardi, K.M.; Brandon, C.A.; Neiswanger, K.; Resick, J.M.; Martin, R.A.; Marazita, M.L. Face shape of unaffected parents with cleft affected offspring: combining three-dimensional surface imaging and geometric morphometrics. Orthod. Craniofac. Res. 2009, 12, 271-281.

35. Albert, A.M.; Greene, D.L. Bilateral asymmetry in skeletal growth and maturation as an indicator of environmental stress. Am. J. Phys. Anthropol. 1999, 110, 341-349.

36. Zaidel, D.W.; Hessamian, M. Asymmetry and symmetry in the beauty of human faces. Symmetry 2010, 2, 136-149.

37. Klingenberg, C.P.; Barluenga, M.; Meyer, A. Shape analysis of symmetric structures: quantifying variation among individuals and asymmetry. Evolution 2002, 56, 1909-1920.

38. Zaidel, D.W.; Aarde, S.M.; Baig, K. Appearance of symmetry, beauty, and health in human faces. Brain Cogn. 2005, 57, 261-263.

39. Ferrario, V.F.; Sforza, C.; Dellavia, C.; Tartaglia, G.M.; Colombo, A.; Carù, A. A quantitative three-dimensional assessment of soft-tissues facial asymmetry of cleft lip and palate adult patients. J. Craniofac. Surg. 2003, 14, 739-746.

40. Bock, M.T.; Bowman, A.W. On the measurement and analysis of asymmetry with applications to facial modelling. J. R. Stat. Soc., Ser. C, Appl. Stat. 2006, 55, 77-91.

41. Kokich, V.O.; Kokich, V.G.; Kiyak, H.A. Perceptions of dental professionals and laypersons to altered dental esthetics: asymmetric and symmetric situations. Am. J. Orthod. Dentofacial Orthop. 2006, 130, 141-151.

42. Kiekens, R.M.; van 't Hof, M.A.; Straatman, H.; Kuijpers-Jagtman, A.M.; Maltha, J.C. Influence of panel composition on aesthetic evaluation of adolescent faces. Eur. J. Orthod. 2007, 29, 95-99.

43. Scott, C.R.; Goonewardene, M.S.; Murray, K. Influence of lips on the perception of malocclusion. Am. J. Orthod. Dentofacial Orthop. 2006, 130, 152-162.

44. Sforza, C.; Ferrario, V.F. Soft-tissue facial anthropometry in three dimensions: from anatomical landmarks to digital morphology in research, clinics and forensic anthropology. J. Anthropol. Sci. 2006, 84, 97-124.

45 De Menezes, M.; Rosati, R.; Ferrario, V.F.; Sforza, C. Accuracy and reproducibility of a 3D stereophotogrammetric imaging system. J. Oral Maxillofac. Surg. 2010, 68, 2129-2135.

46. Hajeer, M.Y.; Ayoub, A.F.; Millett, D.T. Three-dimensional assessment of facial soft-tissue asymmetry before and after orthognathic surgery. Br. J. Oral Maxillofac. Surg. 2004, 42, 396-404.

47. Nkenke, E.; Vairaktaris, E.; Kramer, M.; Schlegel, A.; Holst, A.; Hirschfelder, U.; Wiltfang, J.; Neukam, F.W.; Stamminger, M. Three-dimensional analysis of changes of the malar-midfacial region after LeFort I osteotomy and maxillary advancement. Oral Maxillofac. Surg. 2008, 12, 5-12. 
48. Shi, J.; Samal, A.; Marx, D. How effective are landmarks and their geometry for face recognition? Comput. Vis. Image Underst. 2006, 102, 117-133.

49. Ozsoy, U.; Demirel, B.M.; Yildirim, F.B.; Tosun, O.; Sarikcioglu, L. Method selection in craniofacial measurements: Advantages and disadvantages of 3D digitization method. J. Craniomaxillofac. Surg. 2009, 37, 285-290.

50. Bruner, E.; Bastir, M. Digital Morphology: modelling anatomy and evolution. J. Anthropol. Sci. 2008, 86, 3-5.

51. Ferrario, V.F.; Sforza, C.; Poggio, C.E., Cova, M.; Tartaglia, G. Preliminary evaluation of an electromagnetic three-dimensional digitizer in facial anthropom etry. Cleft Palate Craniofac $J$. 1998, 35, 9-15.

(C) 2010 by the authors; licensee MDPI, Basel, Switzerland. This article is an open access article distributed under the terms and conditions of the Creative Commons Attribution license (http://creativecommons.org/licenses/by/3.0/). 